\title{
Techniques - Robotic-assisted laparoscopic implantation of artificial urinary sphincter with concomitant hysterectomy and sacrocolpopexy
}

Yunwei Zhao; Gary Gray; Blair St. Martin

University of Alberta, Edmonton, AB, Canada

Cite as: Can Urol Assoc J 2018 November 20; Epub ahead of print. http://dx.doi.org/10.5489/cuaj.5580

Published online November 20, 2018

$* * *$

\section{Introduction}

The artificial urinary sphincter (AUS) was first described by Foley in $1943 .{ }^{1}$ The current generation model of AMS 800 (American Medical Systems, MN, U.S.) has been implanted since 1982. Indications for implantation of an AUS include postprostatectomy incontinence, neurogenic bladder dysfunction, intrinsic sphincter deficiency (ISD), and rare congenital causes of incontinence. ${ }^{2}$

When looking specifically at female non-neurogenic stress urinary incontinence, recent studies demonstrate good long-term functional outcomes from the abdominal approach, with success rates of up to $94.4 \%{ }^{3}$ Recent advances in minimally invasive surgery have mitigated the risks of abdominal surgery with the first laparoscopic implantation of AUS published in $2005 .{ }^{4}$ The introduction of robotic-assisted laparoscopic (RAL) surgery brings distinct benefits of superior visualization, improved dexterity, and minimization of blood loss during deep pelvic

dissection. ${ }^{5}$ Hence, we set out to evaluate the role of robotic assistance in AUS implantation in a neurogenic bladder patient with concomitant surgery for pelvic organ prolapse (POP).

\section{Case report}

This patient is a 45-year-old female with persistent urinary incontinence. Initial urodynamic studies demonstrated stress urinary incontinence and she was unsuccessfully treated with an autologous fascial sling and subsequent periurethral bulking agent injection. Repeat urodynamic studies demonstrated absent sensation on filling cystometry and decreased detrusor pressure on pressure flow study. She required daily clean intermittent catheterizations (CIC) to reduce the amount of leakage. Her diagnosis of neurogenic bladder dysfunction was confirmed when she underwent spinal surgery for cauda equina syndrome later that year. On exam, she had grade 3 POP and was consented for combined RAL AUS implantation with hysterectomy and sacrocolpopexy. 


\section{Technique}

The patient received intravenous antibiotics and was given a general anesthetic. She was placed in dorsal lithotomy position. Under Trendelenburg position, direct entry was made using a 12mm disposable trocar supra-umbilically. Two 8mm robotic ports were placed on the left; a third robotic port and a $12 \mathrm{~mm}$ assistant port were placed on the right. After parallel docking of the da Vinci Surgical System (Intuitive Surgical, CA, USA), robotic monopolar scissors, bipolar grasper, and ProGrasp ${ }^{\mathrm{TM}}$ forceps were inserted.

The operation began with RAL bilateral salpingectomy, right oophorectomy, hysterectomy, and sacrocolpopexy. The techniques of RAL sacrocolpopexy have been previously described. ${ }^{6}$ Entry into the pre-vesical space was made by incising the parietal peritoneum between the medial umbilical ligaments. The bladder neck was freed from perivesical fat and the endopelvic fascia at this level was sharply incised laterally. The vaginal surgeon who is experienced in the implantation of AUS introduced two fingers in the vagina to direct placement of the robotic instrument just below the urethra. Circumferential dissection of the urethrovaginal space was accomplished progressively with ProGrasp ${ }^{\mathrm{TM}}$ forceps from both sides of the bladder neck (Fig. 1). Cystoscopy was performed to confirm mucosal integrity. The tape measurer was introduced via the assistant port to measure the size of the cuff and was exchanged with the pressurized cuff. The pressure regulating balloon was introduced into the pre-vesical space via a separate suprapubic incision (Fig. 2). The control pump was introduced to the labia majora with blunt dissection. All tubing connections were made at the suprapubic site and buried subcutaneously. The peritoneum overlying the pre-vesical space was closed with absorbable barbed sutures. The sphincter mechanism was cycled then deactivated.

\section{Results}

Estimated blood loss was minimal. There were no intraoperative complications. Total operative time was $215 \mathrm{~min}$. AUS implantation duration was 99min including 20min dedicated to the circumferential dissection of the bladder neck.

The patient was discharged home on postoperative day (POD) 1 . She required a $14 \mathrm{~F}$ indwelling catheter for 14 days given her neurogenic bladder dysfunction and continued with daily CIC after catheter removal. AUS was activated at 6 weeks postoperatively. At 3 months, she did not require any incontinence pads.

\section{Discussion}

AUS has been used as an effective device for women with severe, refractory incontinence for several decades. There is prospective data demonstrating long term success of $94.4 \%$ after 10 years of follow-up from an abdominal approach in women with ISD. ${ }^{3}$ Laparoscopic and RAL approaches have been introduced only recently but preliminary results show that functional outcomes mirror those of abdominal approaches, albeit with significantly less morbidities. Success rates, when defined as social continence (1 pad/day or less), approach $100 \%$ in the largest RAL series. ${ }^{7}$ Peyronnet et al. compared perioperative outcomes between abdominal and 
RAL implantation of AUS and found a statistically lower postoperative complication rate and a trend toward lower intraoperative complications, decreased blood loss, and shorter lengths of hospitalization in the RAL group. ${ }^{8}$

The most difficult step of the procedure is the development of the urethrovaginal plane due to the absence of a natural plane between the bladder and vagina, especially with scarring from previous anti-incontinence surgeries. ${ }^{2}$ Circumferential dissection of the bladder neck can cause perforations into the bladder, urethra, or vagina, which are known risk factors of sphincter erosion and device explantation. ${ }^{9}$ In RAL series, intraoperative injury and explantation rates approach $40 \%$ and $30 \%$, respectively. ${ }^{7}$ It is crucial to have a surgeon experienced in AUS implantation providing vaginal guidance to the robotic surgeon. Robotic assistance is helpful by providing superior 3-D visualization and orientable instruments. We found that this step alone took 20min to accomplish safely while another series found that it can take up to $66 \mathrm{~min}$. $^{10}$ Compared to other series, our duration of implantation was shorter at $99 \mathrm{~min}$, which could be attributed to our 2-surgeon approach. Our patient was also safely discharged home on POD 1, by taking advantage of the minimally invasive nature of the procedure. Earlier discharge and shorter hospital stay may provide justification for the high costs associated with robotic surgery. Finally, our report presented a patient with neurogenic bladder dysfunction and hence the prolonged catheterization and continuation of CIC postoperatively were therefore expected.

\section{Conclusion}

This report is the first published RAL implantation of AUS in Canada and demonstrates that it can be safely and efficiently performed with other pelvic procedures in a minimally invasive fashion, while providing the patient with benefits of shorter hospital stay and functional continence at 3 months. 


\section{References}

1. Foley FE. An AUS: A new device and operations for the control of enuresis and urinary incontinence. J Urol 1947;58:250.

2. Hussain M, Greenwell, TJ, Venn SN, et al. The current role of the artificial urinary sphincter for the treatment of urinary incontinence. J Urol 2005;174:418-24.

3. Costa P, Poinas G, Ben Naoum K, et al. Long-term results of artificial urinary sphincter for women with type III stress urinary incontinence. Eur Urol 2013;63:753-8.

4. Ngninkeu BN, van Heugen G, di Gregorio M, et al. Laparoscopic artificial urinary sphincter in women for type III incontinence: Preliminary results. Eur Urol 2005;47:7937.

5. Nimeh T, Elliott S. Minimally Invasive Techniques for Bladder Reconstruction. Curr Urol Rep 2018;19:39.

6. Jong K, Klein T, Zimmern PE. Long-term outcomes of robotic mesh sacrocolpopexy. $J$ Robot Surg 2017;4:4.

7. Biardeau X, Rizk J, Marcelli F, et al. Robot-assisted laparoscopic approach for artificial urinary sphincter implantation in 11 women with urinary stress incontinence: Surgical technique and initial experience. Eur Urol 2015;67:937-42.

8. Peyronnet B, Vincendeau S, Tondut L, et al. Artificial urinary sphincter implantation in women with stress urinary incontinence: Preliminary comparison of robot-assisted and open approaches. Int Urogynecol J 2016:27:475-81.

9. Roupret M, Misrai V, Vaessen C, et al. Laparoscopic approach for artificial urinary sphincter implantation in women with intrinsic sphincter deficiency incontinence: a single-centre preliminary experience. Eur Urol 2010;57:499-505.

10. Fournier G, Callerot P, Thoulouzan M, et al. Robotic-assisted laparoscopic implantation of artificial urinary sphincter in women with intrinsic sphincter deficiency incontinence: Initial results. Urology 2014;84:1094-8. 


\section{Figure and Tables}

Fig.1. Circumferential dissection of the bladder neck. The urethrovaginal space was first incised sharply with monopolar scissors and the plane was developed blunted with progressive expansions using robotic ProGrasp ${ }^{\mathrm{TM}}$ forceps.

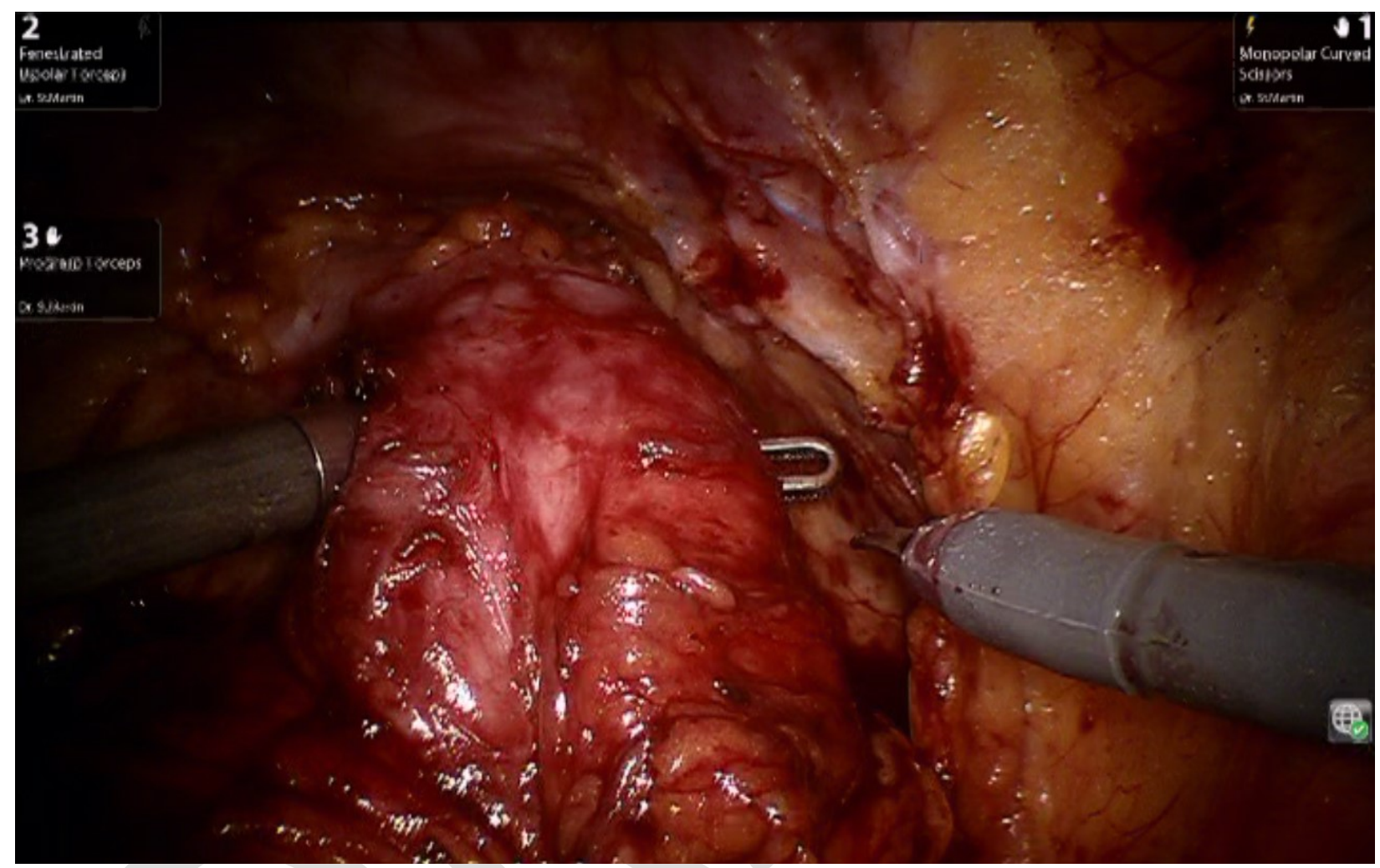


Fig. 2. Placement of the cuff and pressure regulating balloon in the pre-vesical space. A $7 \mathrm{~cm}$ cuff was placed around the bladder neck after it was measured with a tape measurer. A 61-70 cm $\mathrm{H}_{2} \mathrm{O}$ pressure regulating balloon was introduced into the pre-vesical space via a separate suprapubic incision.

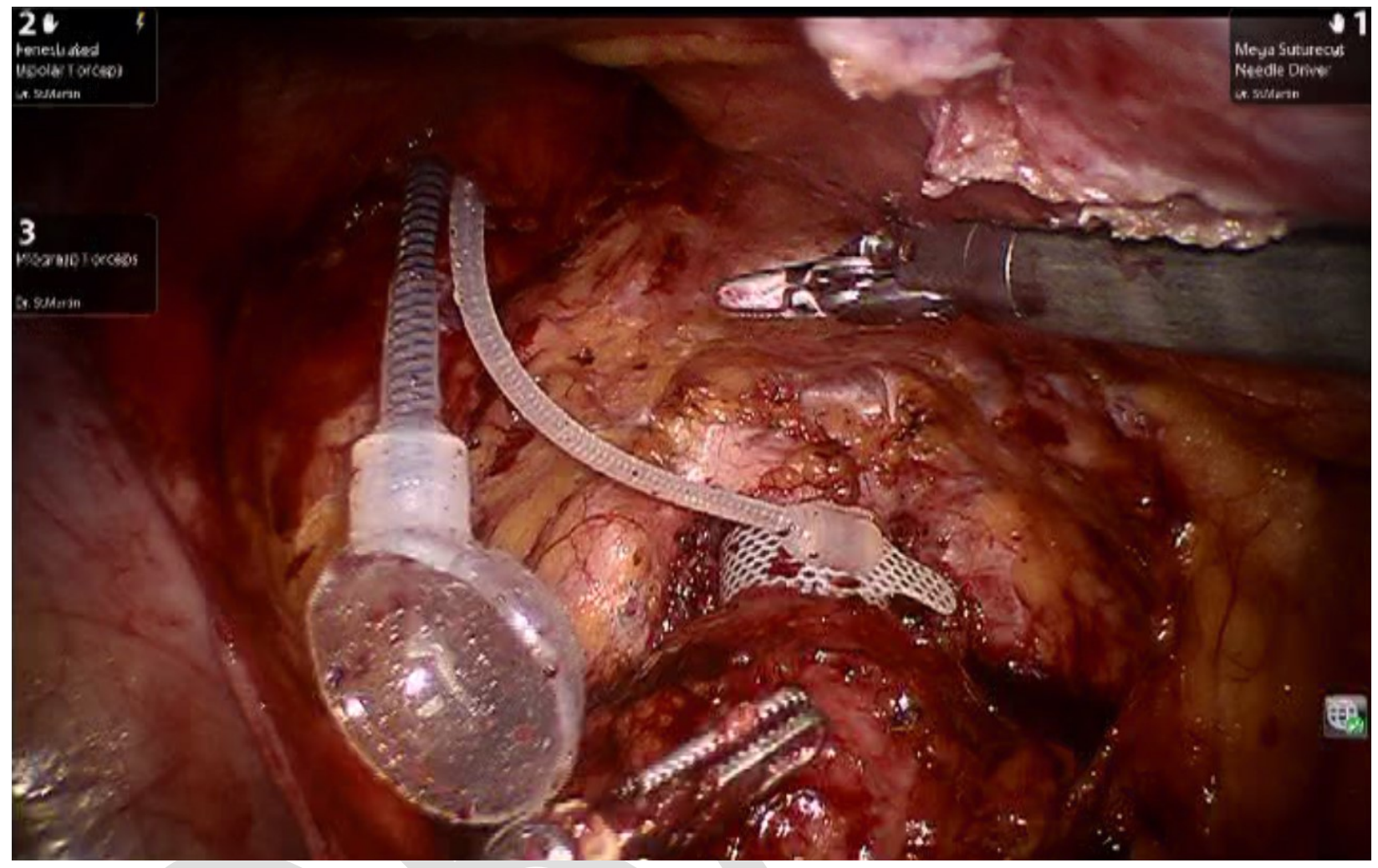

